\title{
Molecular epidemiology of Mycobacterium africanum in Ghana
}

\author{
Adwoa Asante-Poku1 ${ }^{1,2,3}$, Isaac Darko Otchere', Stephen Osei-Wusu', Esther Sarpong ${ }^{1}$, Akosua Baddoo ${ }^{4}$, \\ Audrey Forson ${ }^{4}$, Clement Laryea ${ }^{5}$, Sonia Borrell ${ }^{2,3}$, Frank Bonsu ${ }^{6}$, Jan Hattendorf ${ }^{3,7}$, Collins Ahorlu', \\ Kwadwo A. Koram', Sebastien Gagneux ${ }^{2,3}$ and Dorothy Yeboah-Manu ${ }^{1 *}$ (D)
}

\begin{abstract}
Background: Mycobacterium africanum comprises two phylogenetic lineages within the M. tuberculosis complex (MTBC) and is an important cause of human tuberculosis (TB) in West Africa. The reasons for this geographic restriction of $M$. africanum remain unclear. Here, we performed a prospective study to explore associations between the characteristics of TB patients and the MTBC lineages circulating in Ghana.

Method: We genotyped 1,211 MTBC isolates recovered from pulmonary TB patients recruited between 2012 and 2014 using single nucleotide polymorphism typing and spoligotyping. Associations between patient and pathogen variables were assessed using univariate and multivariate logistic regression.

Results: Of the 1,211 MTBC isolates analysed, $71.9 \%$ (871) belonged to Lineage 4; $12.6 \%$ (152) to Lineage 5 (also known as M. africanum West-Africa 1), 9.2\% (112) to Lineage 6 (also known as M. africanum West-Africa 2) and 0. $6 \%$ (7) to Mycobacterium bovis. Univariate analysis revealed that Lineage 6 strains were less likely to be isoniazid resistant compared to other strains (odds ratio $=0.25,95 \%$ confidence interval $(C \mathrm{l})$ : $0.05-0.77, P<0.01$ ). Multivariate analysis showed that Lineage 5 was significantly more common in patients from the Ewe ethnic group (adjusted odds ratio (adjOR): $2.79 ; 95 \%$ Cl: 1.47-5.29, $P<0.001$ ) and Lineage 6 more likely to be found among HIV-co-infected TB patients (adjOR $=2.2 ; 95 \%$ confidence interval $(C \mathrm{Cl}$ 1.32-3.7, $P<0.001$ ).

Conclusion: Our findings confirm the importance of M. africanum in Ghana and highlight the need to differentiate between Lineage 5 and Lineage 6, as these lineages differ in associated patient variables.
\end{abstract}

\section{Background}

Tuberculosis (TB) remains one of the main global public health problems, particularly in resource-limited settings [1]. With an estimated 9 million new cases annually, and a pool of two billion latently infected individuals, control efforts are struggling in most parts of the world. The TB problem is further exacerbated by a strong synergy with Human immunodeficiency virus infection and acquired immune deficiency syndrome (HIV/AIDS), which is a particularly big challenge in sub-Saharan Africa. Moreover, the global emergence of drug resistance increasingly complicates TB control. The Word Health Organization (WHO) estimates that in 2013, of the 1.1 million TB cases

\footnotetext{
* Correspondence: Dyeboah-Manu@noguchi.ug.edu.gh

${ }^{1}$ Noguchi Memorial Institute for Medical Research, University of Ghana, Legon, Ghana

Full list of author information is available at the end of the article
}

co-infected with HIV, $80 \%$ occurred in Africa, making Africa the hardest hit of the two epidemics [1].

Human TB is mainly caused by Mycobacterium africanum (MAF) and Mycobacterium tuberculosis sensu stricto (MTB), both members of Mycobacterium tuberculosis complex (MTBC), which also includes several sub-species adapted to a variety of wild and domestic animals [2, 3]. MAF first identified in 1968 in Senegal, was initially described biochemically as an intermediary between MTB and M. bovis [4]. Like MTB, MAF strains were found to be sensitive to pyrazinamide; like $M$. bovis, they tended to be a weak producer of niacin, microaerophilic, and unable to reduce nitrate to nitrite [5]. Furthermore, similar to M. bovis they are unable to use glycerol as a sole carbon source due to the lack of functional pyruvate kinase [6]. Initial biochemical features subdivided MAF into two separate groups; the West-African sub-species and East-African also referred 
to as MAF type 1 and 2 [7]. However, based on genomic deletion analyses $[2,8,9]$, we now know that MAF type 2 variant is a sub-lineage of MTB Lineage 4, which has been reclassified as MTB "Uganda sublineage" [10]. MAF type 1 or the "true" $M$. africanum consists of two phylogenetically distinct lineages that differ in their geographic distribution: Lineage 5 (also known as MAF West African 1) is found in the eastern part of WestAfrica, and Lineage 6 (also known as West-Africa genotype II) is mainly found in the western part of WestAfrica. A few countries like Ghana and Cote d'Ivoire harbor both Lineages 5 and $6[9,11]$.

The reason why MAF is largely restricted to the West African region remains unknown, but one possibility might be that MAF is adapted to distinct human population (s) in West Africa [9]. In support of this hypothesis, using a limited retrospective collection of MTBC isolates from South-western Ghana, we recently reported an association between MAF and the Ewe ethnic group [12]. One of the limitations of that previous study was that due to lack of relevant data, we could not control for possible confounding by HIV co-infection. Hence, we followed-up on this initial observation with a larger population-based prospective study in which we included more detailed clinical data and TB cases from both the southern and northern part of Ghana.

\section{Methods}

\section{Ethical Statement and Patient inclusion criteria}

The Institutional Review Board of the Noguchi Memorial Institute for Medical Research (NMIMR) and the Ethikkommission Beider Basel (EKBB) in Basel, Switzerland approved the study and its protocols. Following informed consent, consecutive sputum smear-positive TB cases were recruited from September 2012 to April 2014 from all TB diagnostic centres in the Accra Metropolitan (Southern) and the Mamprusi East (Northern Region) Health Administrations. The standard procedure for sputum sample collection as outlined by the National Tuberculosis Program (NTP) for routine diagnosis of TB in Ghana was used in this study. Participants provided written consent unless the participant was illiterate; in which case witnessed oral consent was used. In accordance with ethical review board regulation in Ghana consent was sought from guardians of children below the age of 18 before enrolment into the study and in some cases child assent was also sought. Samples were taken only after a detailed explanation of the study aims and written or thumb-printed consent obtained for participation. Only newly diagnosed smear-positive, pulmonary TB patients before initiation of treatment or less than 4 weeks of treatment were enrolled into the study. All eligible TB patients were encouraged to undergo HIV testing before initiation of anti-TB treatment according to national guidelines.
Data collected from enrolled patients included age, sex, immunosuppressive co-morbidity with HIV, place of work, previous episode of TB, housing type, place of residence, ethnicity, smoking, level of education, income, and presence of Bacillus Calmette-Guerin (BCG) scar. All patients and staff involved with the study were blinded to the final data obtained and none of the authors have a conflict of interest in the study.

\section{Laboratory tests}

The sputum samples were re-examined for the presence of acid-fast bacilli (AFBs) at NMIMR and cultured on solid agar, after which isolate deoxyribonucleic acid (DNA) was extracted for genotyping analysis. Briefly, we took a $10 \mu \mathrm{L}$ loop full from a Lowenstein-Jensen medium slope and, after heat killing, extracted DNA first by digestion with lysozyme and proteinase $\mathrm{K}$, solubilized by detergents sodium dodecyl sulphate and cetrimonium bromide, followed by chloroform isopropanol extraction [13].

\section{Genotyping of MTBC isolates}

Classification of mycobacterial isolates into the main phylogenetic lineages within the MTBC were by TaqMan real-time PCR (TaqMan, Applied Bio systems, USA) using probes targeting lineage-specific SNPs as reported by Stucki et al. [14]. Spoligotyping was performed to define the sub-lineages and strain families within each of the main lineages circulating in Ghana [15]. Spoligotyping patterns were defined according to SITVITWEB database [16] (http://www.pasteur-guadeloupe.fr: 8081/ SITVIT_ONLINE). SITVITWEB assigned shared types numbers were used whenever a Spoligotyping pattern was found in the database while families and subfamilies were assigned based on the MIRU-VNTRplus database (http: //www. miru-vntrplus.org) [17].

\section{Anti-TB Drug Susceptibility Testing}

All MTBC isolates were screened for their susceptibility to isoniazid (INH) and Rifampicin (RIF) using the Genotype MTBDRplus (Hain lifescience), according to the manufacturer's protocol [18]. Briefly, drug resistance was expressed as the absence of wild-type band, presence of mutation band or both.

\section{Data entry, management and analysis}

Information from the structured questionnaire was double entered using Microsoft Access and validated to remove duplicates and data entry inconsistencies. Spoligotype patterns were entered in a binary format. A series of univariate and multivariable logistic regression models were fitted to assess the relationship between MTBC lineage (s) (primary independent variable) and host variables. For the MTBDRplus assay, definition of specific drug resistance was based on mutations within the katG 
gene and inhA promoter for INH and rifampicin resistance determining region of the rpoB gene for RIF. This was captured as either absence of specific wild-type band and/or presence of mutation band corresponding to specific mutations.

\section{Results}

\section{Patient characteristics}

From September 2012 to April 2014, 1,330 smearpositive TB cases recruited from various diagnostic laboratories in the Greater Accra Region and the Northern Region of Ghana were enrolled into the study. We excluded 80 cases from further analysis because no MTBC cultures were obtained from their sputa. A further 30 strains identified as non-tuberculous mycobacteria and 9 strains which produced ambiguous genotyping results were excluded, leaving 1,211 unique MTBC patient isolates for further analyses.

A summary of characteristics of the study population is provided in Table 1 . The median age of patients was 39 years (range 3 to 91 years). Thirty-one percent (373/ 1,211 ) were females with median age of 33 years; the remaining 838 (69\%) were males with a median age of 36 . Only $2.3 \%(28 / 1,211)$ were children (age $<16$ years). Most of the patients $(N=1,112 ; 91.8 \%)$ were recruited from the southern part of Ghana with the remaining 99 (8.2\%) from northern Ghana. Most patients $(N=1,160$, $95.8 \%)$ were Ghanaians with the remaining 51 (4.2\%) of other West African nationalities. Participants were predominantly of the Akan ethnicity $(N=604,49.9 \%)$, followed by Ga $(N=280,23.1 \%)$, Ewe $(N=184,15.2 \%)$ with the remaining ethnicities $(N=143)$ accounted for $11.8 \%$. Majority of participants $(N=766,63.3 \%)$ had formal education level of secondary education or lower whilst 346 patients $(28.6 \%)$ had no formal education. More than half of our study population $(N=591$, $48.8 \%$ ) were unskilled labourers, 314 skilled (26\%) with the remaining $306(25.2 \%)$ unemployed. Most of the study participants harbored a high bacterial burden as measured by sputum smear microscopy; $3+(N=534$, $44.1 \%)$, followed by $2+(N=295,24.4 \%), 1+(N=266$, $22 \%)$ and scanty $(N=115,9.5 \%)$. All 1,211 TB patients consented to HIV testing, and among them, 160 (13.2 \%) were HIV sero-positive.

\section{The population structure of the MTBC in Ghana}

SNP-typing identified 871 (71.9\%) isolates as Lineage 4 (also known as the Euro American lineage), 152 isolates $(12.6 \%)$ as Lineage 5,112 isolates $(9.2 \%)$ as Lineage 6 , 15 isolates $(1.2 \%)$ as Lineage 1,42 isolates $(3.5 \%)$ as Lineage 2, 12 isolates (1\%) as Lineage 3, and 7 (0.6\%) as $M$. bovis (Additional file 1: Table S1). Among the 871 Lineage 4 isolates, spoligotyping revealed that $503 / 871$ (58 \%) belonged to the 'Cameroon family', the most
Table 1 Characteristics of patients included in the study

\begin{tabular}{|c|c|c|}
\hline Variable & $N($ Total $=1211)$ & $\%$ \\
\hline \multicolumn{3}{|l|}{ Sex } \\
\hline Male & 838 & 69.0 \\
\hline Female & 373 & 31.0 \\
\hline \multicolumn{3}{|l|}{ Age category } \\
\hline yr 08-25 & 227 & 18.7 \\
\hline yr 26-40 & 496 & 41.0 \\
\hline yr 41-77 & 488 & 40.3 \\
\hline \multicolumn{3}{|l|}{ Residency } \\
\hline North & 99 & 8.2 \\
\hline South & 1112 & 91.8 \\
\hline \multicolumn{3}{|l|}{ Occupation } \\
\hline Unskilled & 591 & 48.8 \\
\hline Skilled & 314 & 26.0 \\
\hline Unemployed & 306 & 25.2 \\
\hline \multicolumn{3}{|l|}{ Nationality } \\
\hline Ghanaian & 1160 & 95.8 \\
\hline West Africans & 51 & 4.2 \\
\hline \multicolumn{3}{|l|}{ Income (GHC) } \\
\hline None & 449 & 37.1 \\
\hline$<100$ & 184 & 15.2 \\
\hline $100-500$ & 505 & 41.7 \\
\hline$>500$ & 73 & 6.0 \\
\hline \multicolumn{3}{|l|}{ Smear positivity } \\
\hline Scanty 1-9 & 115 & 9.5 \\
\hline+1 & 266 & 22.0 \\
\hline+2 & 295 & 24.4 \\
\hline+3 & 534 & 44.1 \\
\hline \multicolumn{3}{|l|}{ Ethnicity } \\
\hline Akan & 604 & 49.9 \\
\hline Ewe & 184 & 15.2 \\
\hline Ga & 280 & 23.1 \\
\hline other & 143 & 11.8 \\
\hline \multicolumn{3}{|l|}{ HIV status } \\
\hline Positive & 160 & 13.2 \\
\hline \multicolumn{3}{|l|}{ Education } \\
\hline No Education & 346 & 28.6 \\
\hline Primary & 134 & 11.1 \\
\hline Secondary & 632 & 52.2 \\
\hline Tertiary & 99 & 8.2 \\
\hline \multicolumn{3}{|l|}{ Presence of BCG Scar } \\
\hline Yes & 505 & 41.7 \\
\hline
\end{tabular}

dominant sub-lineage of Lineage 4 in Ghana, with the most prevalent spoligotype 61 accounting for 349 (40.1 \%) isolates. Seventy-three percent of strains 
isolated from other West African nationals (37/51) belonged to the 'Cameroon family'.

In addition to the Cameroon family, seven other sublineages were identified among Lineage 4 based on spoligotyping; Ghana $(N=198 / 871,22.7 \%)$, Haarlem $(N=83$, $9.5 \%)$, Uganda I $(N=27,3.0 \%)$, LAM $(N=26,2.9 \%)$, Uganda II $(N=2,0.2 \%), \mathrm{S}(N=2(0.2 \%)$, New_1 $(N=1$, $0.1 \%)$ and $\mathrm{H} 37 \mathrm{Rv}$ like $(N=1,0.1 \%)$.

Spoligotyping of the 264 MAF (Lineage 5 and 6 combined) isolates revealed 92 distinct spoligotypes patterns. Fifty-two unique patterns (singletons) were observed with the remaining 40 patterns grouped into clusters of 2-26 isolates respectively (Additional file 1: Table S1). Within the 940 MTB isolates, we had 101 patterns, consisting of 60 patterns grouped into clusters of 2-349 isolates respectively, 19 singletons and remaining 11 isolates identified as orphans. We compared the 193 spoligotypes found in this study with those contained in an international spoligotype database, 130 of our spoligotypes were already described in SITVIT database. The other 63 spoligotypes were novel.

\section{Prevalence of drug resistance among the main MTBC lineages}

After excluding the $7 \mathrm{M}$. bovis isolates, all remaining 1,204 isolates were analysed by GenoType ${ }^{\circ}$ MTBDRplus using mutations as specified by manufacturer (Additional file 2: Table S2). Overall, MTBDRplus identified 90/1,204 (7.5 \%), 7/1,204 (0.6\%) and 21/1,211 (1.7\%) of the isolates as INH-mono-resistant, RIF mono-resistant and MDR, respectively (Table 2). Among our data set, the number of isolates belonging to the Lineages $1-3$ was limited. Therefore, for the remaining analyses, we compared Lineage 5 $(N=152)$ and Lineage $6(N=112)$ with all the other MTB lineages combined $(N=940$, Lineages $1-4)$. Based on univariate analysis, we did not find any association between MDR and Lineage 5 compared to Lineages 1-4 (OR 1.1, 95 \% CI: 0.6-2.0), but Lineage 6 was four times less likely to harbour any form of INH resistance $(\mathrm{OR}=0.2595 \%$ CI: $0.05-0.77$, Fischer's Exact test $P<0.01)$. All the RIF mono-resistant isolates $(N=7)$ belonged to Lineage 4 .

\section{MTBC lineage associations with patient characteristics}

Further univariate analyses revealed that Lineage 5 was significantly associated with Ewe ethnicity $(\mathrm{OR}=3.0$,

Table 2 Prevalence of drug resistance among the main MTBC lineages

\begin{tabular}{lccl}
\hline Drug & \multicolumn{3}{l}{ Lineage (Number of Isolates) } \\
\cline { 2 - 4 } Resistance & Lineage 4 (940) & Lineage 5 (152) & Lineage 6 (112) \\
\hline INH $^{\text {mono }}$ & $74(7.9 \%)$ & $14(7.9 \%)$ & $2(3.6 \%)$ \\
RIF $^{\text {mono }}$ & $7(0.7 \%)$ & $0(0.0 \%)$ & $0(0.0 \%)$ \\
MDR & $15(1.6 \%)$ & $6(3.3 \%)$ & $0(0.0 \%)$ \\
\hline
\end{tabular}

95 \% CI: 1.5-4.7) (Table 3). Similarly, Lineage 6 was significantly associated with HIV co-infection $(\mathrm{OR}=2.4$, 95 \% CI: 1.4-3.9). Both of these associations remained statistically significant after adjusting for age and gender using multivariate logistic modeling, (adjusted odds ratio $(\operatorname{adjOR})=2.79,95 \%$ CI: $1.47-5.29$, and $\operatorname{adjOR}=2.2$, $95 \%$ CI: $1.32-3.7$, respectively). No other significant association was found between MTBC lineage and other patient variables, including age, income, sex, the presence of a BCG scar, or the degree of smear positivity.

\section{Discussion and conclusions}

Our molecular epidemiological analysis revealed that i) MAF remains an important pathogen in Ghana, causing one fifth of all human TB cases in Ghana, ii) TB patients of the Ewe ethnicity were more likely to be infected with Lineage 5, and iii) Lineage 6 isolates were less likely to be INH-resistant and more frequent in TB patients coinfected with HIV.

The observation that MAF remains an important cause of pulmonary $\mathrm{TB}$ in Ghana is in contrast to the decline of MAF that has been reported in Cameroon [19]. In that country, MAF might gradually being outcompeted by more virulent MTB. In support for this hypothesis, MAF has been associated with reduced virulence in animal models [4], and a longer latency and a slower rate of progression to active disease in humans [9]. The reason for the persistence of MAF in Ghana over time is unclear but might be partially related to our previous finding that MAF is associated with the Ewe ethnic group [12]. Lineage 5, a finding that we replicated in the present study, drove this association. While we were not able to control for HIV infection in our previous study, in the present study, the patient HIV status was available and included in the analysis. Yet despite this potential confounding factor, the association between Lineage 5 and Ewe ethnicity remained significant. Based on these findings, we hypothesize that Lineage 5 is particularly well adapted to infect and cause TB in the Ewe population [12].

This hypothesis follows on an increasing body of evidence indicating that different MTBC genotypes might be adapted to different human populations [20-23]. Moreover, several studies have reported interactions between host and pathogen genotypes in human TB [22-24]. For example, a study in Vietnam found an association between the T597C allele of the Toll-like receptor 2 gene (TLR2) and infection by Lineage 2 [25]. Similarly, studies performed in Ghana have reported human polymorphism in 5-lipoxygenase (ALOX5) [26] and Mannose Binding Lectin (MBL) which gives protection to TB caused by either MAF or MTB [27]. Most recently, a study in South Africa reported on the association between different HLA class I types and disease caused by different MTBC strain 
Table 3 Distribution of patient variables across the three main MTBC lineages in Ghana

\begin{tabular}{|c|c|c|c|c|c|c|c|}
\hline \multirow[t]{2}{*}{ Risk factor } & \multirow{2}{*}{$\begin{array}{l}\text { MAF Lineage } 5 \\
(N=152) \\
N(\%\end{array}$} & \multirow{2}{*}{$\begin{array}{l}\text { MAF Lineage } 6 \\
(N=112) \\
N(\%\end{array}$} & $\begin{array}{l}\text { MTB (Lineages } \\
1-4)(N=940)\end{array}$ & \multirow[t]{2}{*}{$\begin{array}{l}\text { OR }(95 \% \mathrm{Cl}) \\
\text { (Lineage } 5 \text { vs. 1-4) }\end{array}$} & \multirow[t]{2}{*}{$\begin{array}{l}\text { adjOR }(95 \% \mathrm{Cl})^{\mathrm{a}} \\
(\text { Lineage } 5 \text { vs 1-4) }\end{array}$} & \multirow[t]{2}{*}{$\begin{array}{l}\text { OR }(95 \% \text { Cl) } \\
\text { (Lineage } 6 \text { vs 1-4) }\end{array}$} & \multirow[t]{2}{*}{$\begin{array}{l}\text { adjOR }\left(95 \% \mathrm{Cl}^{\mathrm{a}}\right. \\
(\text { Lineage } 6 \text { vs 1-4) }\end{array}$} \\
\hline & & & \multirow{2}{*}{\multicolumn{5}{|c|}{ N $(\%$}} \\
\hline \multicolumn{3}{|l|}{ Sex } & & & & & \\
\hline Male & $96(63.2)$ & $73(65.2)$ & $662(70.6)$ & \multirow[t]{2}{*}{$1.3(0.97-1.98)$} & & & \\
\hline Female & $56(36.8)$ & 39 (34.8) & $278(29.4)$ & & & & \\
\hline \multicolumn{8}{|l|}{ Age category } \\
\hline yr 08-25 & $28(18.0)$ & $17(15.2)$ & $181(19.4)$ & $1.42(0.82-2.64)$ & & & \\
\hline yr 26-40 & $62(41.0)$ & 49 (43.8) & $383(40.6)$ & $0.96(0.4-1.96)$ & & & \\
\hline yr 41-77 & $62(41.0)$ & $46(41.0)$ & $376(40.0)$ & $1.06(0.59-1.91)$ & & & \\
\hline \multicolumn{8}{|l|}{ Residency } \\
\hline North & $8(5.3)$ & $8(7.1)$ & $82(9.0)$ & $0.58(0.27-1.22)$ & & & \\
\hline South & $144(94.7)$ & $104(92.2)$ & $858(91.0)$ & ref & & & \\
\hline \multicolumn{8}{|l|}{ Occupation } \\
\hline Skilled & $35(23.0)$ & $24(21.4)$ & $171(27.2)$ & $0.9(0.81-1.0)$ & & & \\
\hline Unskilled & $117(77.0)$ & 88 (78.6) & 769 (72.8) & ref & & & \\
\hline \multicolumn{8}{|l|}{ Income (GHC) } \\
\hline None & $65(42.7)$ & $43(38.4)$ & $260(47.3)$ & $0.8(0.62-0.98)^{*}$ & & & \\
\hline$<100$ & $32(21.1)$ & $16(14.3)$ & $136(14.5)$ & $0.91(0.76-1.0)$ & & & \\
\hline $100-500$ & $51(33.5)$ & $45(40.2)$ & $408(32.3)$ & ref & & & \\
\hline$>500$ & $4(2.6)$ & $8(7.1)$ & $58(5.9)$ & $1.3(0.97-1.98)$ & & & \\
\hline \multicolumn{8}{|l|}{ Smear positivity } \\
\hline Scanty 1-9 & $2(1.3)$ & 0 & $80(9.2)$ & $0.6(0.45-0.72)^{*}$ & & & \\
\hline+1 & $34(22.6)$ & $18(16.1)$ & $199(22.8)$ & $0.78(0.65-1.80)$ & & & \\
\hline+2 & $37(24.3)$ & $28(25.0)$ & $213(24.6)$ & $0.98(0.42-1.84)$ & & & \\
\hline+3 & $79(52.0)$ & $66(58.9)$ & $352(40.4)$ & ref & & & \\
\hline \multicolumn{8}{|l|}{ Ethnicity } \\
\hline Akan & $39(25.7)$ & 78 (69.7) & $482(51.4)$ & ref & & & \\
\hline Ewe & $58(38.2)$ & $10(8.9)$ & $116(12.6)$ & $3.0(1.5-4.70)$ & $2.79(1.47-5.29)^{* *}$ & & \\
\hline $\mathrm{Ga}$ & 47 (30.9) & $11(9.8)$ & $221(22.6)$ & & $0.85(0.43-1.69)$ & & \\
\hline Other & $8(5.3)$ & $13(11.6)$ & $118(13.3)$ & & $1.64(0.53-5.34)$ & & \\
\hline \multicolumn{8}{|l|}{ HIV status } \\
\hline Positive & $28(29.5)$ & $29(34.9)$ & $96(18.5)$ & & & $2.4(1.4-3.90)^{*}$ & $2.2(1.32-3.70)^{*}$ \\
\hline \multicolumn{8}{|l|}{ Education } \\
\hline No Education & $50(32.9)$ & $40(35.7)$ & $231(26.5)$ & $0.83(0.75-1.0)$ & & & \\
\hline Primary & $21(13.8)$ & $15(13.4)$ & $91(10.4)$ & $0.72(0.65-1.11)$ & & & \\
\hline Secondary & $70(46.1)$ & $47(42.0)$ & $477(54.8)$ & $0.96(0.83-1.30)$ & & & \\
\hline Tertiary & $11(7.2)$ & $10(8.9)$ & $72(8.3)$ & & & & \\
\hline \multicolumn{8}{|l|}{ BCG Scar } \\
\hline Present & $64(42.1)$ & $46(41.1)$ & $430(41.4)$ & $1.1(0.69-1.7)$ & & & \\
\hline \multicolumn{8}{|l|}{ Smoking } \\
\hline Yes & $25(16.4)$ & $28(25.0)$ & $248(20.6)$ & $0.6(0.7-1.8)$ & & & \\
\hline
\end{tabular}

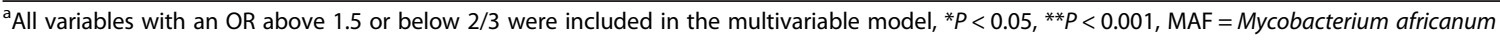
MTB mycobacterium tuberculosis sensu stricto 
families in a South African indigenous population [28]. More studies are needed to confirm the hypothesis that the genetic background of Ewe populations is particularly conducive to infection and disease caused by Lineage 5 and to identify the biological processes involved in this interaction.

Even if the association between Lineage 5 and Ewe ethnicity is contributing to the stable prevalence of MAF in Ghana over time, about half of all MAF cases are due to Lineage 6, which is phylogenetically distinct from Lineage 5 [29]. The findings reported here indicate that Lineage 5 and Lineage 6 also differ phenotypically with respect to their associations with particular patient variables. We found that Lineage 6 was less likely to show any form of INH resistance. This is consistent with reports from Mali that found Lineage 6 less likely to be multidrug-resistant compared to other MTBC lineages [30]. Drug resistance in many bacteria, including MTBC is often associated with a fitness cost [31]. Hence, the fact that Lineage 6 is already attenuated compared to other MTBC lineages $[9,32]$ might limit its potential for drug resistance acquisition. In support of this idea, Lineage 6 has been associated with inhA promoter mutations conferring low levels of resistance to isoniazid that have no known impact on bacterial fitness [33]. In contrast, most other MTBC strains tend to harbour mutations in $k a t G$ conferring high levels of resistance, some of which are associated with a high fitness cost [34].

The relative attenuation of Lineage 6 has led to the hypothesis that this lineage might be an opportunistic pathogen [35]. This view is supported by the findings reported here, as well as previous work by others [9] showing an association between Lineage 6 and HIV coinfection [34]. A previous study from Ghana found no such association, perhaps reflecting differences in study design [36]. Moreover, de Jong et al. reported that Lineage 6 was significantly more frequent in elderly TB patients, consistent with both slower progression to active disease [37] and the role of age-related immunosuppression [34]. Recent advances in phylogenomics of the MTBC show that even though Lineage 6 has primarily been isolated from humans, it is phylogenetically most closely related to several animal-adapted variants of the MTBC [24] including the recently discovered chimpanzee bacillus [38] as well as $M$. suricattae and $M$. mungi which cause TB-like disease in meerkats and banded mangooses, respectively [39]. The potential for an unknown animal reservoir for Lineage 6 has been discussed for some time but so far, none has been discovered [9]. Moreover, unlike other animal-adapted members of the MTBC that rarely cause TB in humans and cannot maintain a full life cycle in human populations [40], Lineage 6 transmits readily among humans [9]. So far, the role of HIV in driving Lineage 6 remains unclear.
Even though HIV had a dramatic impact on the TB epidemic in Sub-Saharan Africa, Lineage 6 remains restricted to the Western part of the continent, which shows much lower rates of HIV infection compared to Southern Africa. Hence, other factors are likely to determine to epidemiology of Lineage 6.

In conclusion, our study confirms that MAF remains an important cause of human TB in Ghana. Our findings also emphasize the need to differentiate between Lineage 5 and 6, and show that the differences between these lineages are relevant for understanding the phylogeography of MAF; while human genetic factors may drive Lineage 5, unknown environmental factors seem to influence the epidemiology of Lineage 6. More generally, these results also highlight the current problems with the species nomenclature in the MTBC [39]. Indeed, the phylogenetic, phenotypic, and ecological differences between Lineage 5 and Lineage 6 argue against them being collectively referred to as MAF, as opposed to just separate lineages within MTB. Finally, given the current efforts in the development of new $\mathrm{TB}$ vaccines, our findings also highlight the need to consider the MTBC variability in the development of new tools and strategies to control TB especially in area where Lineages 5 and 6 are prevalent.

\section{Additional files}

Additional file 1: Table S1. Interpretation of MTBDRplus results with specific mutations. (DOC $43 \mathrm{~kb}$ )

Additional file 2: Table S2. Genotyping profile of 1211 MTBC isolates from Ghana. (DOC 577 kb)

Abbreviations

AFB, acid fast bacilli; AIDS, acquired immune deficiency syndrome; BCG, bacillus calmette-guerin; DNA, deoxyribonucleic acid; EKBB, ethikkommission beider basel; HIV, human immunodeficiency virus infection; INH, isoniazid; $M A F$, mycobacterium africanum; MBL, mannose binding lectin; MDR, multi drug resistance; $M T B$, mycobacterium tuberculosis; $M T B C$, mycobacterium tuberculosis complex; NMIMR, noguchi memorial institute for medical research; NTP, national tuberculosis programme; RIF, rifampicin; TB, tuberculosis; TLR, toll like receptor

\section{Acknowledgements}

We express our gratitude to all laboratory staff and study participants of the various health facilities for their time and cooperation during the study period. We also acknowledge Prof Douglas Young, NIMR, UK and Prof Jakob Zinsstag SwissTPH for useful discussions.

\section{Funding}

This study was supported by the Wellcome Trust grant 097134/Z/11/Z to DYM. Funder had no role in study design, data collection and analysis, decision to publish, or preparation of the manuscript.

Availability of data and materials

All the data supporting our findings is contained within the manuscript and will be shared upon request.

Authors' contributions

DYM, AAP, KK, AF, FB and SG: Conceived and designed study. DYM, AAP and SG: Wrote the first draft manuscript. AAP, IDO, JH: Analyzed data. AAP, IDO, 
SOW, ES: Performed the experiments. AB, AF, CL, CA and SB: Case Recruitment and Acquisition of Data. All authors read and approved manuscript

\section{Competing interests}

The authors declare that they have no competing interest.

\section{Consent for publication}

Not applicable.

\section{Ethics approval and consent to participate}

This study was approved by the Institutional Review Board of the Noguch Memorial Institute for Medical Research (NMIMR) and the Ethikkommission Beider Basel (EKBB) in Basel, Switzerland. Participants provided written consent unless the participant was illiterate; in which case witnessed oral consent was used. In accordance with ethical review board regulation in Ghana consent was sought from guardians of children below the age of 18 before enrolment into the study and in some cases child assent was also sought.

\section{Author details}

'Noguchi Memorial Institute for Medical Research, University of Ghana, Legon, Ghana. ${ }^{2}$ Department of Medical Parasitology and Infection Biology, Swiss Tropical and Public Health Institute, Basel, Switzerland. ${ }^{3}$ University of Basel, Basel, Switzerland. ${ }^{4}$ Department of Chest Diseases, Korle-Bu Teaching Hospital, Korle-bu, Accra, Ghana. ${ }^{5} 37$ Military Hospital, Accra, Ghana. ${ }^{6}$ National Tuberculosis Programme, Ghana health Service, Accra, Ghana. ${ }^{7}$ Department of Epidemiology and Public Health, Swiss Tropical and Public Health Institute, Basel, Switzerland.

\section{Received: 1 December 2015 Accepted: 20 July 2016} Published online: 09 August 2016

\section{References}

1. World Health Organization. World Health Organization Global Tuberculosis Report. Geneva: World Health Organization; 2014.

2. Brosch R, Gordon SV, Marmiesse M, Brodin P, Buchrieser C, Eiglmeier K, et al. A new evolutionary scenario for the Mycobacterium tuberculosis complex. Proc Natl Acad Sci U S A. 2002;99:3684-9.

3. Gagneux S, Small PM. Global phylogeography of Mycobacterium tuberculosis and implications for tuberculosis product development. Lancet Infect Dis. 2007;7:328-37

4. Castets $\mathrm{M}$, Boisvert $\mathrm{H}$, Grumbach F, Brunel M, Rist N. Tuberculosis bacilli of the African type: preliminary note]. Revue de tuberculose et de pneumologie. 1968:32:179-84.

5. Pattyn SR, Portaels F, Spanoghe L, Magos J. Further studies on African strains of Mycobacterium tuberculosis: comparison with $M$. bovis and $M$. microti. Ann Soc Belges Med Trop Parasitol Mycol. 1970;50:211-27.

6. Keating LA, Wheeler PR, Mansoor H, Inwald JK. The pyruvate requirement of some members of the Mycobacterium tuberculosis complex is due to an inactive pyruvate kinase: implications for in vivo growth. Mol Microbiol. 2005;56:163-74.

7. David HL, Jahan MT, Jumin A, Grandry J, Lehman EH. Numerical taxonomy analysis of Mycobacterium africanum. Int J Syst Bacteriol. 1978;28:464-72

8. Mostowy S, Onipede A, Gagneux S, Niemann S, Kremer K, Desmond EA, et al. Genomic analysis distinguishes Mycobacterium africanum. J Clin Microbiol. 2004;42:3594-9.

9. De Jong BC, Antonio M, Gagneux S. Mycobacterium africanum-Review of an Important Cause of Human Tuberculosis in West Africa. PLoS Negl Trop Dis. 2010:4, e744.

10. Wampande EM, Mupere E, Debanne SM, Asiimwe BB, Nsereko M, Mayanja $\mathrm{H}$, et al. Long-term dominance of Mycobacterium tuberculosis Uganda family in peri-urban Kampala-Uganda is not associated with cavitary disease. BMC Infect Dis. 2013;13:484

11. Yeboah-Manu D, Asante-Poku A, Bodmer T, Stucki D, Koram K, Bonsu F, et al. Genotypic diversity and drug susceptibility patterns among M. tuberculosis complex isolates from South-Western Ghana. PLOS ONE. 2011;6:e21906.

12. Asante-Poku A, Yeboah-Manu D, Otchere ID, Aboagye SY, Stucki D, Hattendorf J, et al. Mycobacterium africanum is Associated with Patient Ethnicity in Ghana. PLoS Negl Trop Dis. 2015;9, e3370.

13. Van Soolingen D, De Hass EW, Hermans PW, Groenen PM, Van Embden JD. Comparison of various repetitive DNA elements as genetic markers for strain differentiation and epidemiology of Mycobacterium tuberculosis. J Clin Microbiol. 1993:31:1987.

14. Stucki D, Malla B, Hostettler S, Huna T, Feldmann J, Yeboah-Manu D, et al. Two new rapid SNP-typing methods for classifying Mycobacterium tuberculosis complex into the main phylogenetic lineages. PLoS ONE. 2012;7, e41253.

15. Kamerbeek J, Schouls L, Kolk A, Van Agterveld M, Van Soolingen D, Kuijper S, et al. Simultaneous detection and strain differentiation of Mycobacterium tuberculosis for diagnosis and epidemiology. J Clin Microbiol. 1997;35:907-14.

16. Demay C, Liens B, Burguiere T, Hill V, Couvin D, Millet J, et al. SITVITWEB-a publicly available international multimarker database for studying Mycobacterium tuberculosis genetic diversity and molecular epidemiology. Infect Genet Evol. 2012;12:755-66.

17. Weniger T, Krawczyk J, Supply P, Niemann S, Harmsen D. MIRU-VNTRplus: a web tool for polyphasic genotyping of Mycobacterium tuberculosis complex bacteria. Nucleic Acids Res. 2010;38.

18. Marinus B, Heidi A, Gerrit C, Obrien R, Bosman ME. Rapid Molecular Screening for Multidrug-Resistant Tuberculosis in a High-Volume Public Health Laboratory in South Africa. Am J Respir Crit Care Med. 2008;177:787-92.

19. Niobe-Eyangoh SN, Kuaban C, Sorlin P, Cunin P, Thonnon J, Sola C, et al. Genetic biodiversity of Mycobacterium tuberculosis complex strains from patients with pulmonary tuberculosis in Cameroon. J Clin Microbiol. 2003:41:2547-53.

20. Fenner L, Egger M, Bodmer T, Furrer $H$, Ballif $M$, Helbling $P$, et al. HIV infection disrupts the sympatric host-pathogen relationship in human tuberculosis. PLoS Genet. 2013;9, e1003318.

21. Pasipanodya JG, Moonan PK, Veino E, Miller TL, Fernandez M, Slocum P, et al. Allopatric tuberculosis host-pathogen relationships are associated with greater pulmonary impairment. Infect Genet Evol. 2013;16:433-40.

22. Gagneux S. Host-pathogen coevolution in human tuberculosis. Philosophical Transactions of the Royal Society B: Biological Sciences. 2012;367:850-9.

23. Brites D, Gagneux S. Co-evolution of Mycobacterium tuberculosis and Homo sapiens. Immunol Rev. 2015;264:6-24.

24. Coscolla M, Gagneux S. Does M. tuberculosis genomic diversity explain disease diversity? Drug discovery today. Disease mechanisms. 2010;7:e43-59.

25. Caws M, Thwaites G, Dustan S, Hawn TR, Nguyen TNL, Nguyen TTT, et al. The influence of host and bacterial genotype on the development of disseminated disease with Mycobacterium tuberculosis. PLoS Pathog. 2008:4, e1000034

26. Herb F, Thye T, Niemann S, Browne ENL, Chinbuah MA, Gyapong J, et al. ALOX5 variants associated with susceptibility to human pulmonary tuberculosis. Hum Mol Genet. 2008;17:1052-60.

27. Thye T, Niemann S, Walter K, Homolka S, Intemann CD, Intemann CD, et al. Variant G57E of Mannose Binding Lectin associated with protection against tuberculosis caused by Mycobacterium africanum but not by $\mathrm{M}$. tuberculosis. PLoS ONE. 2011;6:e2090.

28. Salie M, van der Merwe L, Moller M, Daya M, van der Spuy GD, Van Helden PD, et al. Associations between human leukocyte antigen class I variants and the Mycobacterium tuberculosis subtypes causing disease. J Infect Dis. 2014:209:216-23.

29. Coscolla M, Gagneux S. Consequences of genomic diversity in Mycobacterium tuberculosis. Semin Immunol. 2014:26:431-44.

30. Traore B, Diarra B, Dembele BPP, Somboro AM, Hammond AS, Siddiqui S, et al. Molecular strain typing of Mycobacterium tuberculosis complex in Bamako, Mali. Int J Tuberc Lung Dis. 2012;16:911-6.

31. Borrell S, Gagneux S. Infectiousness, reproductive fitness and evolution of drugresistant Mycobacterium tuberculosis. Int J Tuberc Lung Dis. 2009;13:1456-66.

32. Bentley SD, Comas I, Bryant JM, Walker D, Smith NH, Harris SR, et al. The Genome of Mycobacterium Africanum West African 2 Reveals a LineageSpecific Locus and Genome Erosion Common to the M. tuberculosis Complex. PLoS Negl Trop Dis. 2012;6, e1552.

33. Homolka S, Meyer CG, Hilleman D, Owusu-Dabo E, Adjei O, Horstmann RD, et al. Unequal distribution of resistance-conferring mutations among Mycobacterium tuberculosis and Mycobacterium africanum strains from Ghana. Int J Med Microb. 2010:300:489-95.

34. Fenner L, Egger M, Bodmer T, Altpeter E, Zwahlen M, Jaton K, et al. Effect of Mutation and Genetic Background on Drug Resistance in Mycobacterium tuberculosis. Antimicrob. Agents Chemother. 2010;56:3047-53.

35. De Jong BC, Hill PC, Brookes RH, Otu JK, Peterson KL, Small PM, et al. Mycobacterium africanum: a new opportunistic pathogen in HIV infection? AIDS. 2005:19:1714-5.

36. Meyer CG, Scarisbrick S, Niemann S, Browne EN, Chinbuah MA, Gyapang J, et al. Pulmonary tuberculosis: Virulence of Mycobacterium africanum and relevance in HIV co-infection. Tuberculosis. 2008;88:482e489. 
37. De Jong BC, Hill PC, Aiken A, Awine T, Antonio M, Adetifa IM, et al. Progression to Active Tuberculosis, but Not Transmission, Varies by Mycobacterium tuberculosis Lineage in The Gambia. J Infect Dis. 2008;198:1037-10.

38. Coscolla M, Lewin A, Metzger S, Calvignac-Spencer S, Nitsche A, et al. Novel Mycobacterium tuberculosis Complex Isolate from a Wild Chimpanzee. Emerg Infect Dis. 2013;19:969-76.

39. Parsons SDC, Drewe JA, Gey van Pittius NC, Warren RM, Van Helden PD. Novel Cause of Tuberculosis in Meerkats, South Africa. Emerg Infect Dis. 2013:19:2004-7.

40. Berg S, Schelling E, Hailu E, Firedessa R, Gumi B, Erenso G, et al. Investigation of the high rates of extrapulmonary tuberculosis in Ethiopia reveals no single driving factor and minimal evidence for zoonotic transmission of Mycobacterium bovis infection. BMC Infect Dis. 2015;15:112.

Submit your next manuscript to BioMed Central and we will help you at every step:

- We accept pre-submission inquiries

- Our selector tool helps you to find the most relevant journal

- We provide round the clock customer support

- Convenient online submission

- Thorough peer review

- Inclusion in PubMed and all major indexing services

- Maximum visibility for your research

Submit your manuscript at www.biomedcentral.com/submit
Biomed Central 